\title{
New challenges for the Journal of Echocardiography with an innovative system for online submission, editorial processing, and publication
}

\author{
Yutaka Otsuji
}

Published online: 22 January 2009

(C) Japanese Society of Echocardiography 2009

The Journal of Echocardiography, the official publication of the Japanese Society of Echocardiography, was launched in 2003. At that time the founding editor-in-chief, Kiyoshi Yoshida, announced that the journal would publish original investigations, review articles, case reports, letters to the editor, editorials, and images in cardiovascular medicine in the field of echocardiography from Japan, Asia, and all other parts of the world. That purpose was achieved through the publication of many articles with clinical, educational, and investigative value.

I recently had the privilege of taking over the chief editor's role from Dr. Yoshida, beginning in January 2009. I would like to emphasize that as members of the Japanese Society of Echocardiography, we have a mission to meet the challenges of making the Journal of Echocardiography even more attractive as it plays its important role in cardiovascular ultrasound. This field is expanding rapidly, with a growing number of patients who require echocardiographic evaluations. Novel echocardiographic technologies are rapidly being developed with increasing needs for validation and clinical applications. I expect that patients will welcome the challenges we have set for ourselves in the Journal of Echocardiography.

I am pleased to announce that new systems for online submission, editorial processing, and publication have been put into effect by our publisher, Springer, beginning with this issue of the journal. For submissions, the online system makes possible easier access, reduces the work required for editorial processing, and shortens the time from submission to publication. It also provides quick online publication in advance of the print publication shortly after receipt of corrected proofs. Because dynamic images are essential in echocardiography, I encourage authors to submit not only conventional still-frame figures but also illustrative videos, which can be published online. I believe that these features of our new publication system will significantly enhance the value of the Journal of Echocardiography.

I invite all physicians, sonographers, researchers, and others who work in echocardiography for the benefit of patients to join in meeting the new challenges presented to us in the Journal of Echocardiography.
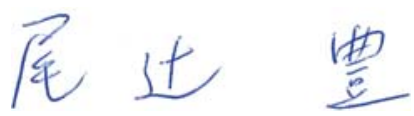

Yutaka Otsuji, MD Editor-in-Chief

Y. Otsuji ( $\square)$

Second Department of Internal Medicine,

University of Occupational and Environmental Health,

1-1 Iseigaoka, Yahatanishi-ku,

Kitakyushu 807-8555, Japan

e-mail: otsujiy@med.uoeh-u.ac.jp 